\title{
Asymptotic Behavior of the Newton-Boussinesq Equation in a Two-Dimensional Channel
}

\author{
Guglielmo Fucci \\ Department of Physics \\ New Mexico Institute of Mining and Technology, Socorro, NM 87801, USA \\ Bixiang Wang,$\quad$ Preeti Singh \\ Department of Mathematics \\ New Mexico Institute of Mining and Technology, Socorro, NM 87801, USA
}

\begin{abstract}
We prove the existence of a global attractor for the Newton-Boussinesq equation defined in a two-dimensional channel. The asymptotic compactness of the equation is derived by the uniform estimates on the tails of solutions. We also establish the regularity of the global attractor.
\end{abstract}

Key words. Newton-Boussinesq equation, global attractor, asymptotic compactness.

MSC 2000. Primary 35B40. Secondary 35B41, 37L30.

\section{Introduction}

In this paper, we investigate the asymptotic behavior of solutions of the Newton-Boussinesq equation defined in an unbounded domain. Let $\Omega=(0, L) \times \mathbb{R}$ where $L$ is a positive number. Consider the system of equations defined in $(x, y) \in \Omega$ and $t>0$ :

$$
\begin{gathered}
\partial_{t} \xi+u \partial_{x} \xi+v \partial_{y} \xi=\triangle \xi-\frac{R_{a}}{P_{r}} \partial_{x} \theta+f(x, y), \\
\triangle \Psi=\xi, \quad u=\Psi_{y}, \quad v=-\Psi_{x},
\end{gathered}
$$

\footnotetext{
${ }^{*}$ Supported in part by the Start-up Fund of New Mexico Institute of Mining and Technology.
} 


$$
\partial_{t} \theta+u \partial_{x} \theta+v \partial_{y} \theta=\frac{1}{P_{r}} \triangle \theta+g(x, y),
$$

where $\vec{u}=(u, v)$ is the velocity vector of the fluid, $\theta$ is the flow temperature, $\Psi$ is the flow function, $\xi$ is the vortex. The positive constants $P_{r}$ and $R_{a}$ are the Prandtl number and the Rayleigh number, respectively. The external terms $f$ and $g$ are given in $L^{2}(\Omega)$.

The Newton-Boussinesq equation describes many physical phenomena such as Benard flow, see, [7, 8] and the references therein. If the domain is bounded, the existence, uniqueness and the asymptotic behavior of solutions of system (1.1)-(1.3) have been studied by several authors, see, e.g., [9, 10, 11, 12]. In this paper we will examine the dynamical behavior of the solutions when the system is defined in the unbounded two-dimensional channel $\Omega$. More precisely, we will prove the existence of a global attractor for the system. Note that the unboundedness of the domain $\Omega$ introduces a major difficulty for proving the existence of a global attractor because Sobolev embeddings are no longer compact in this case, and hence the asymptotic compactness of the solution operator cannot be obtained by a standard method. Several approaches have been developed to overcome this difficulty. The energy equation method is one way to prove the asymptotic compactness of equations defined in unbounded domains. This idea was first developed by Ball in [5, 6] to deal with the compactness of the wave equation and the NavierStokes equation in bounded domains, and then extended by other authors in [14, 16, 20] to the Navier-Stokes equation in unbounded domains. Note that the energy equation of the NavierStokes equation in $L^{2}(\Omega)$ does not contain the nonlinear term. This fact together with the weak compactness can be used to prove the strong asymptotic compactness in $L^{2}(\Omega)$ (see, e.g., [16, 20]). However, in our case, the energy equation for system (1.1)-(1.3) in $L^{2}(\Omega)$ does contain the nonlinear term, and hence the energy equation approach does not apply. In this paper, we will employ the techniques of uniform estimates on the tails of solutions to establish the asymptotic compactness of the Newton-Boussinesq equation. This idea was develop in [24] for proving the asymptotic compactness of the Reaction-Diffusion equation in unbounded domains, and later used by several authors in [1, 2, 3, 15, 17, 19, 22].

This paper is organized as follows. In the next section, we derive uniform estimates for the 
solutions of the system (1.1)-(1.3) when $t \rightarrow \infty$, which are necessary for proving the existence of a bounded absorbing set and the asymptotic compactness of the equation. In Section 3, we first establish the asymptotic compactness of system (1.1)-(1.3) by uniform estimates on the tails of solutions, and then prove the existence of a global attractor. The regularity of the global attractor is given in the last section.

In the sequel, we adopt the following notations. The norm of $L^{2}(\Omega)$ is denoted by $\|\cdot\|$ which is defined by mean of the usual inner product $(\cdot, \cdot)$. The norm of any Banach space $X$ is written as $\|\cdot\|_{X}$. In particular $\|\cdot\|_{p}$ represents the norm of $L^{p}(\Omega)$. The letter $C$ is a generic positive constant which may change its value from line to line.

Throughout this paper, we frequently use the following inequality

$$
\|u\|_{4}<C\|u\|_{H^{1}(\Omega)}^{\frac{1}{2}}\|u\|^{\frac{1}{2}}, \quad \forall u \in H^{1}(\Omega),
$$

and the Poincare inequality

$$
\|u\| \leq \lambda\|\nabla u\| \quad \forall u \in H_{0}^{1}(\Omega)
$$

where $\lambda$ is a positive constant.

\section{Uniform Estimates of Solutions}

In this section, we derive uniform estimates for the solutions of the system (1.1)-(1.3) for large time. We also prove that the tails of solutions are uniformly small when space and time variables are sufficiently large.

Notice that system (1.1)-(1.3) can be rewritten as follows: for every $(x, y) \in \Omega$ and $t>0$,

$$
\begin{gathered}
\frac{\partial \xi}{\partial t}-\triangle \xi+J(\Psi, \xi)+\frac{R_{a}}{P_{r}} \frac{\partial \theta}{\partial x}=f(x, y), \\
\triangle \Psi=\xi \\
\frac{\partial \theta}{\partial t}-\frac{1}{P_{r}} \triangle \theta+J(\Psi, \theta)=g(x, y),
\end{gathered}
$$


with the boundary conditions

$$
\left.\xi\right|_{\partial \Omega}=0,\left.\quad \theta\right|_{\partial \Omega}=0,\left.\quad \Psi\right|_{\partial \Omega}=0,
$$

and the initial conditions

$$
\xi(x, y, 0)=\xi_{0}(x, y), \quad \theta(x, y, 0)=\theta_{0}(x, y)
$$

where the functional $J$ is given by

$$
J(u, v)=u_{y} v_{x}-u_{x} v_{y} .
$$

It is easy to verify that $J$ satisfies:

$$
\begin{gathered}
\int_{\Omega} J(u, v) v d x d y=0, \quad \text { for all } \quad u \in H^{1}(\Omega), v \in H^{2}(\Omega) \cap H_{0}^{1}(\Omega), \\
\|J(u, v)\| \leq C\|u\|_{H^{2}}\|v\|_{H^{2}}, \quad \text { for all } u \in H^{2}(\Omega), v \in H^{2}(\Omega), \\
\|J(u, v)\| \leq C\|u\|_{H^{3}}\|\nabla v\|, \quad \text { for all } u \in H^{3}(\Omega), v \in H^{1}(\Omega) .
\end{gathered}
$$

It is standard to prove that problem (2.1) $-(2.5)$ is well posed in $L^{2}(\Omega) \times L^{2}(\Omega)$ (see, e.g., [9]). More precisely, for every $\left(\xi_{0}, \theta_{0}\right) \in L^{2}(\Omega) \times L^{2}(\Omega)$, system (2.1)-(2.5) has a unique solution $(\xi, \theta)$ such that for every $T>0$,

$$
\begin{aligned}
& \xi \in C^{0}\left([0, \infty), L^{2}(\Omega)\right) \cap L^{2}\left(0, T ; H_{0}^{1}(\Omega)\right), \\
& \theta \in C^{0}\left([0, \infty), L^{2}(\Omega)\right) \cap L^{2}\left(0, T ; H_{0}^{1}(\Omega)\right) .
\end{aligned}
$$

Therefore, we can define a semigroup $\{S(t)\}_{t \geq 0}$ such that for every $t \geq 0, S(t)$ maps $L^{2}(\Omega) \times$ $L^{2}(\Omega)$ into itself and $S(t)\left(\xi_{0}, \theta_{0}\right)=(\xi(t), \theta(t))$. We now start to derive uniform estimates for the dynamical system $\{S(t)\}_{t \geq 0}$.

Lemma 2.1. Suppose that $\left(\xi_{0}, \theta_{0}\right) \in L^{2}(\Omega) \times L^{2}(\Omega)$. Then for every $T>0$, there is a constant $C>0$ such that the solution $(\xi, \theta)$ of system (2.1)-(2.5) satisfies

$$
\|\xi(t)\|+\|\theta(t)\| \leq C \quad \forall t \in[0, T]
$$

where $C$ depends only on the data $\left(\Omega, P_{r}, R_{a}\right)$, $T$ depends on the data $\left(\Omega, P_{r}, R_{a}\right)$ and $R$ when $\left\|\xi_{0}\right\| \leq R$ and $\left\|\theta_{0}\right\| \leq R$ 
Proof. We first consider equation (2.3). By taking the inner product of (2.3) with $\theta$ in $L^{2}(\Omega)$ and using relation (2.7) we obtain

$$
\frac{1}{2} \frac{d}{d t}\|\theta\|^{2}+\frac{1}{P_{r}}\|\nabla \theta\|^{2}=\int_{\Omega} g \theta d x d y
$$

By Poincairé inequality (1.5) we find that

$$
\frac{1}{2} \frac{d}{d t}\|\theta\|^{2}++\frac{1}{2 P_{r}}\|\nabla \theta\|^{2}+\frac{1}{2 \lambda^{2} P_{r}}\|\theta\|^{2} \leq \int_{\Omega} g \theta d x d y .
$$

Notice that the right-hand side is bounded by

$$
\int_{\Omega} g \theta d x d y \leq\|g\|\|\theta\| \leq C\|g\|^{2}+\frac{1}{4 \lambda^{2} P_{r}}\|\theta\|^{2}
$$

By (2.10) $-(2.12)$ we find that

$$
\frac{d}{d t}\|\theta\|^{2}+\frac{1}{P_{r}}\|\nabla \theta\|^{2}+\frac{1}{2 \lambda^{2} P_{r}}\|\theta\|^{2} \leq C, \quad \forall t \geq 0
$$

which implies that

$$
\frac{d}{d t}\|\theta\|^{2}+C_{1}\|\theta\|^{2} \leq C, \quad \forall t \geq 0
$$

Let $T>0$ be fixed and take $t \in[0, T]$, integrating (2.14) over $(0, t)$ we obtain

$$
\|\theta(t)\|^{2} \leq C_{1} T+\|\theta(0)\|^{2} \leq C_{1} T+R^{2} \leq C, \quad \forall t \in[0, T]
$$

We now consider equation (2.1). By taking the inner product of (2.1) with $\xi$ in $L^{2}(\Omega)$ and using relation (2.7) we obtain

$$
\frac{1}{2} \frac{d}{d t}\|\xi\|^{2}+\|\nabla \xi\|^{2}+\frac{R_{a}}{P_{r}} \int_{\Omega} \theta_{x} \xi d x d y=\int_{\Omega} f \xi d x d y
$$

We notice that the following inequalities hold

$$
\frac{R_{a}}{P_{r}}\left|\int_{\Omega} \theta_{x} \xi d x d y\right|=\frac{R_{a}}{P_{r}}\left|\int_{\Omega} \xi_{x} \theta d x d y\right| \leq \frac{R_{a}}{P_{r}}\left\|\nabla \xi \left|\||| \theta\| \leq \frac{1}{4}\|\nabla \xi\|^{2}+C\|\theta\|^{2}\right.\right.
$$

and

$$
\left|\int_{\Omega} f \xi d x d y\right| \leq\|f|\||| \xi\| \leq \lambda||f|\||| \nabla \xi\left\|\leq \frac{1}{4}\right\| \nabla \xi \|^{2}+C
$$


By (2.15), (2.17) and (2.18), it follows from (2.16) that

$$
\frac{d}{d t}\|\xi\|^{2}+\|\nabla \xi\|^{2} \leq C, \quad \forall t \in[0, T]
$$

Then Poincairè inequality implies that

$$
\frac{d}{d t}\|\xi\|^{2}+C_{1}\|\xi\|^{2} \leq C, \quad \forall t \in[0, T]
$$

Integrating (2.20) on $(0, t)$ we obtain

$$
\|\xi(t)\|^{2} \leq C T+\|\xi(0)\| \leq C, \quad \forall t \in[0, T] .
$$

Combining (2.15) and (2.21) we conclude that

$$
\|\theta(t)\|+\|\xi(t)\| \leq C, \quad \forall t \in[0, T] .
$$

The proof is complete.

Lemma 2.2. Suppose that $\left(\xi_{0}, \theta_{0}\right) \in L^{2}(\Omega) \times L^{2}(\Omega)$. Then for the solution $(\xi, \theta)$ of system (2.1)- (2.5) we have

$$
\|\xi(t)\|+\|\theta(t)\| \leq M_{1} \quad \forall t \geq t_{1}
$$

and

$$
\int_{t}^{t+1}\|\nabla \xi(\tau)\|^{2} d \tau+\int_{t}^{t+1}\|\nabla \theta(\tau)\| d \tau \leq M_{2} \quad \forall t \geq t_{1}
$$

where $M_{1}$ and $M_{2}$ are constants depending only on the data $\left(\Omega, P_{r}, R_{a}\right), t_{1}$ depends on the data $\left(\Omega, P_{r}, R_{a}\right)$ and $R$ when $\left\|\xi_{0}\right\| \leq R$ and $\left\|\theta_{0}\right\| \leq R$.

Proof. By (2.14) and Gronwall inequality we infer that

$$
\|\theta(t)\|^{2} \leq e^{-C_{1} t}\|\theta(0)\|^{2}+C_{2} \leq e^{-C_{1} t} R^{2}+C_{2} \leq 2 C_{2} \quad \forall t \geq t_{1}^{*},
$$

where $t_{1}^{*}=\frac{1}{C_{1}} \ln \left(\frac{R^{2}}{C_{2}}\right)$. Moreover by (2.17), (2.18) and (2.23) we get from (2.20) that

$$
\frac{d}{d t}\|\xi\|^{2}+C_{1}\|\xi\|^{2} \leq C, \quad \forall t \geq t_{1}^{*}
$$


By Lemma 2.1 and Gronwall inequality we have

$$
\begin{aligned}
\|\xi(t)\|^{2} & \leq e^{-C_{1}\left(t-t_{1}^{*}\right)}\left\|\xi\left(t_{1}^{*}\right)\right\|^{2}+\frac{C}{C_{1}} \\
& \leq e^{-C_{1}\left(t-t_{1}^{*}\right)} C_{2}+\frac{C}{C_{1}} \leq \frac{2 C}{C_{1}}, \quad \forall t \geq t_{2}^{\star}+\frac{1}{C_{1}} \ln \left(\frac{C_{1} C_{2}}{C}\right),
\end{aligned}
$$

Combining (2.23) and (2.25) we find that

$$
\|\theta(t)\|+\|\xi(t)\| \leq C, \quad \forall t \geq t_{1}
$$

where $t_{1}=\max \left\{t_{1}^{*}, t_{2}^{*}\right\}$. By (2.13) we obtain that

$$
\frac{d}{d \tau}\|\theta\|^{2}+C\|\nabla \theta\|^{2} \leq C_{1}, \quad \forall \tau \geq t_{1} .
$$

Integrating (2.27) on $(t, t+1)$, by (2.26) we have that

$$
\int_{t}^{t+1}\|\nabla \theta(\tau)\|^{2} d \tau \leq C, \quad \forall t \geq t_{1}
$$

By (2.16) and (2.25) we also have

$$
\frac{d}{d \tau}\|\xi\|^{2}+C\|\nabla \xi\|^{2} \leq C_{1}, \quad \forall \tau \geq t_{1} .
$$

Integrating (2.29) on $(t, t+1)$, by (2.26) we get

$$
\int_{t}^{t+1}\|\nabla \xi(\tau)\|^{2} d \tau \leq C, \quad \forall t \geq t_{1}
$$

Then Lemma 2.2 follows from (2.26), (2.28) and (2.30).

We now derive uniform estimates in $H^{1}(\Omega)$.

Lemma 2.3. Suppose that $\left(\xi_{0}, \theta_{0}\right) \in L^{2}(\Omega) \times L^{2}(\Omega)$. Then for the solution $(\xi, \theta)$ of system (2.1)-(2.5) we have

$$
\|\nabla \xi(t)\|+\|\nabla \theta(t)\| \leq M_{3} \quad \forall t \geq t_{3}
$$

and

$$
\int_{t}^{t+1}\left(\|\triangle \xi(t)\|^{2}+\|\triangle \theta(t)\|^{2}\right) d t \leq M_{3}, \quad \forall t \geq t_{3},
$$

where $M_{3}$ is a constant depending only on the data $\left(\Omega, P_{r}, R_{a}\right), t_{3}$ depends on the data $\left(\Omega, P_{r}, R_{a}\right)$ and $R$ when $\left\|\xi_{0}\right\| \leq R$ and $\left\|\theta_{0}\right\| \leq R$. 
Proof. Taking the inner product of (2.1) with $\triangle \xi$ in $L^{2}(\Omega)$ we get

$$
\frac{1}{2} \frac{d}{d t}\|\nabla \xi\|^{2}+\|\triangle \xi\|^{2}=\int_{\Omega} J(\Psi, \xi) \triangle \xi d x d y+\frac{R_{a}}{P_{r}} \int_{\Omega} \theta_{x} \triangle \xi d x d y-\int_{\Omega} f \triangle \xi d x d y .
$$

Notice that the first term on the right-hand side of (2.31) is given by

$$
\int_{\Omega} J(\Psi, \xi) \triangle \xi d x d y=\int_{\Omega} \Psi_{y} \xi_{x} \triangle \xi d x d y+\int_{\Omega} \Psi_{x} \xi_{y} \triangle \xi d x d y .
$$

We now estimate the first term on the right-hand side of (2.32). By (1.4) and Lemma 2.2 we have the following estimates for $t \geq T$,

$$
\begin{gathered}
\int_{\Omega} \Psi_{y} \xi_{x} \triangle \xi d x d y \leq\left\|\Psi_{y}\right\|_{4}\left\|\xi_{x}\right\|_{4}\|\triangle \xi\| \leq C\left\|\Psi_{y}\right\|^{\frac{1}{2}}\left\|\Psi_{y}\right\|_{H^{1}}^{\frac{1}{2}}\left\|\xi_{x}\right\|^{\frac{1}{2}}\left\|\xi_{x}\right\|_{H^{1}}^{\frac{1}{2}}\|\triangle \xi\| \\
\leq C\|\Psi\|_{H^{2}}\|\nabla \xi\|^{\frac{1}{2}}\|\triangle \xi\|^{\frac{3}{2}} \leq C\|\nabla \xi\|^{\frac{1}{2}}\|\triangle \xi\|^{\frac{3}{2}} \leq \frac{1}{8}\|\triangle \xi\|^{2}+C\|\nabla \xi\|^{2} .
\end{gathered}
$$

Similarly for the second term on the right-hand side of (2.32) we have

$$
\int_{\Omega} \Psi_{x} \xi_{y} \triangle \xi d x d y \leq \frac{1}{8}\|\triangle \xi\|^{2}+C\|\nabla \xi\|^{2}
$$

It follows from (2.32) and (2.34) that

$$
\int_{\Omega} J(\Psi, \xi) \triangle \xi d x d y \leq \frac{1}{4}\|\triangle \xi\|^{2}+C\|\nabla \xi\|^{2} .
$$

Note that the last two terms on the right-hand side of (2.31) are bounded by

$$
C\left|\int_{\Omega} \theta_{x} \triangle \xi d x d y\right|+\left|\int_{\Omega} f \triangle \xi d x d y\right| \leq \frac{1}{4}\|\triangle \xi\|^{2}+C\|\nabla \theta\|^{2}+C .
$$

From (2.31) and (2.35)-(2.36) we have

$$
\frac{d}{d t}\|\nabla \xi\|^{2}+\|\triangle \xi\|^{2} \leq C\left(\|\nabla \xi\|^{2}+\|\nabla \theta\|^{2}\right)+C, \quad \forall t \geq T .
$$

Taking the inner product of (2.3) with $\triangle \theta$ we get

$$
\frac{1}{2} \frac{d}{d t}\|\nabla \theta\|^{2}+\frac{1}{P_{r}}\|\triangle \theta\|^{2}=\int_{\Omega} J(\Psi, \theta) \triangle \theta d x d y+\int_{\Omega} g \triangle \theta d x d y .
$$

By arguments similar to (2.35) and (2.36), we obtain that

$$
\frac{d}{d t}\|\nabla \theta\|^{2}+\frac{1}{2 P_{r}}\|\triangle \theta\|^{2} \leq C\left(\|\nabla \xi\|^{2}+\|\nabla \theta\|^{2}\right)+C, \quad \forall t \geq T .
$$


Let $\alpha=\min \left\{1, \frac{1}{2 P_{r}}\right\}$. Then from (2.37) and (2.39), we have

$$
\frac{d}{d t}\left(\|\nabla \xi\|^{2}+\|\nabla \theta\|^{2}\right)+\alpha\left(\|\triangle \theta\|^{2}+\|\triangle \xi\|^{2}\right) \leq C\left(\|\nabla \xi\|^{2}+\|\nabla \theta\|^{2}\right)+C, \quad \forall t \geq T .
$$

By the uniform Gronwall inequality and Lemma 2.2, we find from (2.40) that

$$
\|\nabla \xi(t)\|+\|\nabla \theta(t)\| \leq C, \quad \forall t \geq T+1
$$

Integrating (2.40) on $(t, t+1)$, by (2.41) we get

$$
\int_{t}^{t+1}\left(\|\triangle \xi(t)\|^{2}+\|\triangle \theta(t)\|^{2}\right) d t \leq C, \quad \forall t \geq T+1
$$

Then Lemma 2.3 follows from (2.41) $-(2.42)$.

Next we establish the uniform estimates on the tails of solutions which are crucial for proving the asymptotic compactness of the solution operator. Given $k>0$, we denote by $\Omega_{k}$ the set $\Omega_{k}=\{(x, y) \in \Omega:|y| \leq k\}$ and $\Omega \backslash \Omega_{k}$ the complement of $\Omega_{k}$. For our purpose, we choose a smooth cut-off function $\phi$ such that $0 \leq \phi(s) \leq 1$ and

$$
\begin{cases}\phi(s)=0 & \text { if }|s|<1 \\ \phi(s)=1 & \text { if }|s|>2\end{cases}
$$

Then, we have the following Poincairé type of inequality

Lemma 2.4. Let $v \in H_{0}^{1}(\Omega)$ and $\phi$ be given as above. Then $\exists \alpha>0$ and $\beta>0$ such that $\forall k>0$ :

$$
\int_{\Omega} \phi^{2}\left(\frac{|y|^{2}}{k^{2}}\right)|\nabla v|^{2} d x d y \geq \alpha \int_{\Omega} \phi^{2}\left(\frac{|y|^{2}}{k^{2}}\right) v^{2} d x d y-\frac{\beta}{k^{2}} \int_{\Omega} v^{2} d x d y
$$

Proof. By Poincairè inequality (1.5), we have

$$
\int_{\Omega} \phi^{2}\left(\frac{|y|^{2}}{k^{2}}\right) v^{2} d x d y \leq \lambda^{2} \int_{\Omega}\left|\nabla\left(\phi\left(\frac{|y|^{2}}{k^{2}}\right) v\right)\right|^{2} d x d y
$$

Notice that

$$
\int_{\Omega}\left|\nabla\left(\phi\left(\frac{|y|^{2}}{k^{2}}\right) v\right)\right|^{2} d x d y \leq \int_{\Omega} \phi^{2}\left(\frac{|y|^{2}}{k^{2}}\right)|\nabla v|^{2} d x d y+4 \int_{\Omega}\left(\phi^{\prime}\left(\frac{|y|^{2}}{k^{2}}\right)\right)^{2} \frac{y^{2}}{k^{4}} v^{2} d x d y
$$




$$
\begin{gathered}
\leq \int_{\Omega} \phi^{2}\left(\frac{|y|^{2}}{k^{2}}\right)|\nabla v|^{2} d x d y+4 \int_{k \leq|y| \leq \sqrt{2} k}\left(\phi^{\prime}\left(\frac{|y|^{2}}{k^{2}}\right)\right)^{2} \frac{y^{2}}{k^{4}} v^{2} d x d y \\
\leq \int_{\Omega} \phi^{2}\left(\frac{|y|^{2}}{k^{2}}\right)|\nabla v|^{2} d x d y+\frac{C}{k^{2}} \int_{k \leq|y| \leq \sqrt{2} k}|v|^{2} d x d y \\
\leq \int_{\Omega} \phi^{2}\left(\frac{|y|^{2}}{k^{2}}\right)|\nabla v|^{2} d x d y+\frac{C}{k^{2}} \int_{\Omega}|v|^{2} d x d y .
\end{gathered}
$$

From (2.44) and (2.45) it follows that

$$
\int_{\Omega} \phi^{2}\left(\frac{|y|^{2}}{k^{2}}\right) v^{2} d x d y \leq \lambda^{2} \int_{\Omega} \phi^{2}\left(\frac{|y|^{2}}{k^{2}}\right)|\nabla v|^{2} d x d y+\frac{C}{k^{2}} \int_{\Omega}|v|^{2} d x d y
$$

which implies Lemma 2.4. The proof is complete.

Lemma 2.5. Given $\epsilon>0$, then there exist $t_{3}>0$ and $k_{0}>0$ such that the solution $(\xi, \theta)$ of system (2.1)-(2.5) with the initial condition $\left(\xi_{0}, \theta_{0}\right)$ satisfies

$$
\int_{\Omega \backslash \Omega_{k_{0}}}\left(|\xi(t)|^{2}+|\theta(t)|^{2}\right) d x d y \leq \epsilon, \quad \forall t \geq t_{3}
$$

where $k_{0}$ depends only on the data $\left(\Omega, P_{r}, R_{a}\right)$ and $\epsilon, t_{3}$ depends only on $\left(\Omega, P_{r}, R_{a}\right)$, $\epsilon$ and $R$ when $\left\|\xi_{0}\right\| \leq R$ and $\left\|\theta_{0}\right\| \leq R$.

Proof. Multiplying (2.3) by $\phi^{2}\left(\frac{|y|^{2}}{k^{2}}\right) \theta(x, y, t)$ and then integrating the resulting identity over $\Omega$, we obtain

$$
\begin{aligned}
\frac{1}{2} & \frac{d}{d t} \int_{\Omega}|\theta|^{2} \phi^{2}\left(\frac{|y|^{2}}{k^{2}}\right) d x d y-\frac{1}{P_{r}} \int_{\Omega}(\theta \triangle \theta) \phi^{2}\left(\frac{|y|^{2}}{k^{2}}\right) d x d y \\
& =\int_{\Omega} g \theta \phi^{2}\left(\frac{|y|^{2}}{k^{2}}\right) d x d y-\int_{\Omega} J(\Psi, \theta) \theta \phi^{2}\left(\frac{|y|^{2}}{k^{2}}\right) d x d y .
\end{aligned}
$$

We now estimate every term in (2.47). We first have, by Lemma 2.4,

$$
\begin{gathered}
-\frac{1}{P_{r}} \int_{\Omega}(\theta \triangle \theta) \phi^{2}\left(\frac{|y|^{2}}{k^{2}}\right) d x d y \\
=\frac{1}{P_{r}} \int_{\Omega} \phi^{2}\left(\frac{|y|^{2}}{k^{2}}\right)|\nabla \theta|^{2} d x d y+\frac{4}{P_{r}} \int_{\Omega} \phi\left(\frac{|y|^{2}}{k^{2}}\right) \phi^{\prime}\left(\frac{|y|^{2}}{k^{2}}\right) \theta \theta_{y} \frac{y}{k^{2}} d x d y \\
\geq \frac{\alpha}{P_{r}} \int_{\Omega} \phi^{2}\left(\frac{|y|^{2}}{k^{2}}\right)|\theta|^{2} d x d y-\frac{C}{k^{2}}\|\theta\|^{2}+\frac{4}{P_{r}} \int_{\Omega} \phi\left(\frac{|y|^{2}}{k^{2}}\right) \phi^{\prime}\left(\frac{|y|^{2}}{k^{2}}\right) \theta \theta_{y} \frac{y}{k^{2}} d x d y .
\end{gathered}
$$


For the last term on the right-hand side of (2.47) we obtain, by integration by parts,

$$
\begin{gathered}
\int_{\Omega} J(\Psi, \theta) \theta \phi^{2}\left(\frac{|y|^{2}}{k^{2}}\right) d x d y \\
=\int_{\Omega} \Psi_{y} \theta_{x} \theta \phi^{2}\left(\frac{|y|^{2}}{k^{2}}\right) d x d y-\int_{\Omega} \Psi_{x} \theta \theta \phi^{2}\left(\frac{|y|^{2}}{k^{2}}\right) d x d y \\
=\int_{\Omega} \Psi_{y}\left(\frac{1}{2} \theta^{2}\right)_{x} \phi^{2}\left(\frac{|y|^{2}}{k^{2}}\right) d x d y-\int_{\Omega} \Psi_{x}\left(\frac{1}{2} \theta^{2}\right)_{y} \phi^{2}\left(\frac{|y|^{2}}{k^{2}}\right) d x d y \\
=-\frac{1}{2} \int_{\Omega} \Psi_{y x} \theta^{2} \phi^{2}\left(\frac{|y|^{2}}{k^{2}}\right) d x d y+\frac{1}{2} \int_{\Omega} \Psi_{y x} \theta^{2} \phi^{2}\left(\frac{|y|^{2}}{k^{2}}\right) d x d y \\
+2 \int_{\Omega} \Psi_{x} \theta^{2} \phi^{\prime}\left(\frac{|y|^{2}}{k^{2}}\right) \phi\left(\frac{|y|^{2}}{k^{2}}\right) \frac{y}{k^{2}} d x d y \\
=2 \int_{\Omega} \Psi_{x} \theta^{2} \phi^{\prime}\left(\frac{|y|^{2}}{k^{2}}\right) \phi\left(\frac{|y|^{2}}{k^{2}}\right) \frac{y}{k^{2}} d x d y
\end{gathered}
$$

It follows, from (2.47) through (2.49) that

$$
\begin{aligned}
& \frac{1}{2} \frac{d}{d t} \int_{\Omega}|\theta|^{2} \phi^{2}\left(\frac{|y|^{2}}{k^{2}}\right) d x d y+\frac{\alpha}{P_{r}} \int_{\Omega} \phi^{2}\left(\frac{|y|^{2}}{k^{2}}\right)|\theta|^{2} d x d y \\
= & \int_{\Omega} g \theta \phi^{2}\left(\frac{|y|^{2}}{k^{2}}\right) d x d y-\frac{4}{P_{r}} \int_{\Omega} \phi\left(\frac{|y|^{2}}{k^{2}}\right) \phi^{\prime}\left(\frac{|y|^{2}}{k^{2}}\right) \theta \theta_{y} \frac{y}{k^{2}} d x d y \\
& -2 \int_{\Omega} \Psi_{x} \theta^{2} \phi^{\prime}\left(\frac{|y|^{2}}{k^{2}}\right) \phi\left(\frac{|y|^{2}}{k^{2}}\right) \frac{y}{k^{2}} d x d+\frac{C}{k^{2}}\|\theta\|^{2} .
\end{aligned}
$$

Note that the first term on the right-hand side of (2.50) is bounded by

$$
\begin{gathered}
\left|\int_{\Omega} g \theta \phi^{2}\left(\frac{|y|^{2}}{k^{2}}\right) d x d y\right|=\left|\int_{|y| \geq k} g \theta \phi^{2}\left(\frac{|y|^{2}}{k^{2}}\right) d x d y\right| \\
\leq\left(\int_{|y| \geq k} g^{2} d x d y\right)^{\frac{1}{2}}\left(\int_{|y| \geq k} \phi^{4}\left(\frac{|y|^{2}}{k^{2}}\right) \theta^{2} d x d y\right)^{\frac{1}{2}} \\
\leq\left(\int_{|y| \geq k} g^{2} d x d y\right)^{\frac{1}{2}}\left(\int_{\Omega} \phi^{2}\left(\frac{|y|^{2}}{k^{2}}\right) \theta^{2} d x d y\right)^{\frac{1}{2}} \\
\leq C \int_{|y| \geq k}|g|^{2} d x d y+\frac{\alpha}{2 P_{r}} \int_{\Omega}|\theta|^{2} \phi^{2}\left(\frac{|y|^{2}}{k^{2}}\right) d x d y .
\end{gathered}
$$

For the second term on the right-hand side of (2.50) we have

$$
\frac{4}{P_{r}}\left|\int_{\Omega} \phi\left(\frac{|y|^{2}}{k^{2}}\right) \phi^{\prime}\left(\frac{|y|^{2}}{k^{2}}\right) \theta \theta_{y} \frac{y}{k^{2}} d x d y\right|
$$




$$
\begin{aligned}
& =\frac{4}{P_{r}}\left|\int_{k \leq|y| \leq \sqrt{2 k}} \phi\left(\frac{|y|^{2}}{k^{2}}\right) \phi^{\prime}\left(\frac{|y|^{2}}{k^{2}}\right) \theta \theta_{y} \frac{y}{k^{2}} d x d y\right| \\
& \leq \frac{C}{k} \int_{k \leq|y| \leq \sqrt{2 k}}|\theta|\left|\theta_{y}\right| d x d y \leq \frac{C}{k}\|\theta|||| \nabla \theta\| \leq \frac{C}{k} .
\end{aligned}
$$

where the last inequality is obtained by Lemmas (2.2) and (2.3). The third term on the righthand side is bounded by

$$
\begin{aligned}
& 2\left|\int_{\Omega} \Psi_{x} \theta^{2} \phi^{\prime}\left(\frac{|y|^{2}}{k^{2}}\right) \phi\left(\frac{|y|^{2}}{k^{2}}\right) \frac{y}{k^{2}} d x d y\right| \\
= & 2\left|\int_{k \leq|y| \leq \sqrt{2 k}} \Psi_{x} \theta^{2} \phi^{\prime}\left(\frac{|y|^{2}}{k^{2}}\right) \phi\left(\frac{|y|^{2}}{k^{2}}\right) \frac{y}{k^{2}} d x d y\right| \\
\leq & \frac{C}{k} \int_{k \leq|y| \leq \sqrt{2 k}}\left|\Psi_{x}\right||\theta|^{2} d x d y \leq \frac{C}{k} \int_{\Omega}\left|\Psi_{x} \| \theta\right|^{2} d x d y \\
\leq & \frac{C}{k}\left\|\Psi_{x}\right\|_{6}\|\theta\|_{3}\|\theta\| \leq \frac{C}{k}\|\Psi\|_{H^{2}}\|\theta\|_{H^{1}}\|\theta\| \leq \frac{C}{k} .
\end{aligned}
$$

It follows, from (2.50) through (2.53) that for $k \geq 1$

$$
\frac{d}{d t} \int_{\Omega}|\theta|^{2} \phi^{2}\left(\frac{|y|^{2}}{k^{2}}\right) d x d y+\frac{\alpha}{P_{r}} \int_{\Omega}|\theta|^{2} \phi^{2}\left(\frac{|y|^{2}}{k^{2}}\right) d x d y \leq \frac{C}{k}+C_{1} \int_{|y| \geq k}|g|^{2} d x d y .
$$

Now, since $g \in L^{2}(\Omega)$, given $\epsilon>0$, there exists $k_{1}>0$ such that

$$
C_{1} \int_{|y| \geq k}|g|^{2} d x d y \leq \frac{\epsilon}{2}, \quad \forall k \geq k_{1}(\epsilon)
$$

Let $k_{2}=\max \left\{k_{1}, \frac{2 C}{\epsilon}\right\}$, then by (2.54) and (2.55) we obtain that for all $k \geq k_{2}$ and $t \geq T$,

$$
\frac{d}{d t} \int_{\Omega}|\theta|^{2} \phi^{2}\left(\frac{|y|^{2}}{k^{2}}\right) d x d y+\frac{\alpha}{P_{r}} \int_{\Omega}|\theta|^{2} \phi^{2}\left(\frac{|y|^{2}}{k^{2}}\right) d x d y \leq \epsilon .
$$

Applying Gronwall lemma to (2.56), by Lemma 2.2 we find that, for all $k \geq k_{2}$,

$$
\begin{array}{r}
\int_{\Omega} \phi^{2}\left(\frac{|y|^{2}}{k^{2}}\right)|\theta|^{2} d x d y \leq e^{-\frac{\alpha}{P_{r}}(t-T)} \int_{\Omega} \phi^{2}\left(\frac{|y|^{2}}{k^{2}}\right)|\theta(T)|^{2} d x d y+\frac{\epsilon \alpha}{P_{r}} \\
\leq e^{-\frac{\alpha}{P_{r}}(t-T)}\|\theta(T)\|^{2}+\frac{\epsilon \alpha}{P_{r}} \leq \frac{2 \alpha \epsilon}{P_{r}}
\end{array}
$$


for all $t \geq T_{1}=T-\frac{P_{r}}{\alpha} \ln \left(\frac{\alpha \epsilon}{C P_{r}}\right)$. We now estimate $\int_{\Omega} \phi^{2}\left(\frac{|y|^{2}}{k^{2}}\right)|\xi(x, y, t)|^{2} d x d y$. Multiplying (2.1) by $\phi^{2}\left(\frac{|y|^{2}}{k^{2}}\right) \xi(x, y, t)$ and then integrating by parts we get

$$
\begin{gathered}
\frac{1}{2} \frac{d}{d t} \int_{\Omega}|\xi|^{2} \phi^{2}\left(\frac{|y|^{2}}{k^{2}}\right) d x d y-\int_{\Omega}(\xi \triangle \xi) \phi^{2}\left(\frac{|y|^{2}}{k^{2}}\right) d x d y+\int_{\Omega} J(\Psi, \xi) \xi \phi^{2}\left(\frac{|y|^{2}}{k^{2}}\right) d x d y \\
=\int_{\Omega} f \xi \phi^{2}\left(\frac{|y|^{2}}{k^{2}}\right) d x d y+\frac{R_{a}}{P_{r}} \int_{\Omega} \theta_{x} \xi \phi^{2}\left(\frac{|y|^{2}}{k^{2}}\right) d x d y
\end{gathered}
$$

For the second term on the left-hand side of (2.58), by Lemma 2.4 we have

$$
\begin{gathered}
-\int_{\Omega}(\xi \triangle \xi) \phi^{2}\left(\frac{|y|^{2}}{k^{2}}\right) d x d y \\
=\int_{\Omega}|\nabla \xi|^{2} \phi^{2}\left(\frac{|y|^{2}}{k^{2}}\right) d x d y+4 \int_{\Omega} \xi \xi_{y} \phi\left(\frac{|y|^{2}}{k^{2}}\right) \phi^{\prime}\left(\frac{|y|^{2}}{k^{2}}\right) \frac{y}{k^{2}} d x d y \\
\geq \frac{1}{2} \int_{\Omega} \phi^{2}\left(\frac{|y|^{2}}{k^{2}}\right)|\nabla \xi|^{2} d x d y+\frac{\alpha}{2} \int_{\Omega} \phi^{2}\left(\frac{|y|^{2}}{k^{2}}\right)|\xi|^{2} d x d y \\
-\frac{C}{k^{2}} \|\left.\xi\right|^{2}+4 \int_{\Omega} \xi \xi_{y} \phi\left(\frac{|y|^{2}}{k^{2}}\right) \phi^{\prime}\left(\frac{|y|^{2}}{k^{2}}\right) \frac{y}{k^{2}} d x d y .
\end{gathered}
$$

By (2.58) and (2.59) we find that the following inequality holds

$$
\begin{gathered}
\frac{1}{2} \frac{d}{d t} \int_{\Omega}|\xi|^{2} \phi^{2}\left(\frac{|y|^{2}}{k^{2}}\right) d x d y+\frac{1}{2} \int_{\Omega}|\nabla \xi|^{2} \phi^{2}\left(\frac{|y|^{2}}{k^{2}}\right) d x d y+\frac{\alpha}{2} \int_{\Omega} \phi^{2}\left(\frac{|y|^{2}}{k^{2}}\right)|\xi|^{2} d x d y \\
\leq \int_{\Omega} f \xi \phi^{2}\left(\frac{|y|^{2}}{k^{2}}\right) d x d y-\int_{\Omega} J(\Psi, \xi) \xi \phi^{2}\left(\frac{|y|^{2}}{k^{2}}\right) d x d y+\frac{R_{a}}{P_{r}} \int_{\Omega} \theta_{x} \xi \phi^{2}\left(\frac{|y|^{2}}{k^{2}}\right) d x d y \\
-4 \int_{\Omega} \xi \xi_{y} \phi\left(\frac{|y|^{2}}{k^{2}}\right) \phi^{\prime}\left(\frac{|y|^{2}}{k^{2}}\right) \frac{y}{k^{2}} d x d y+\frac{C}{k^{2}}\|\xi\|^{2} .
\end{gathered}
$$

Note that the third term on the right-hand side of (2.60) is bounded by

$$
\begin{aligned}
& \frac{R_{a}}{P_{r}}\left|\int_{\Omega} \theta_{x} \xi \phi^{2}\left(\frac{|y|^{2}}{k^{2}}\right) d x d y\right| \leq \frac{R_{a}}{P_{r}}\left|\int_{\Omega} \theta \xi_{x} \phi^{2}\left(\frac{|y|^{2}}{k^{2}}\right) d x d y\right| \\
& \quad \leq \frac{1}{2} \int_{\Omega} \xi_{x}^{2} \phi^{2}\left(\frac{|y|^{2}}{k^{2}}\right) d x d y+\frac{R_{a}^{2}}{2 P_{r}^{2}} \int_{\Omega}|\theta|^{2} \phi^{2}\left(\frac{|y|^{2}}{k^{2}}\right) \\
& \quad \leq \frac{1}{2} \int_{\Omega}|\nabla \xi|^{2} \phi^{2}\left(\frac{|y|^{2}}{k^{2}}\right) d x d y+C \epsilon,
\end{aligned}
$$


where the last inequality is obtained by (2.57). It follows, then, from (2.60) and (2.61) that

$$
\begin{array}{r}
\frac{1}{2} \frac{d}{d t} \int_{\Omega}|\xi|^{2} \phi^{2}\left(\frac{|y|^{2}}{k^{2}}\right) d x d y+\frac{\alpha}{2} \int_{\Omega}|\xi|^{2} \phi^{2}\left(\frac{|y|^{2}}{k^{2}}\right) d x d y \\
\leq \int_{\Omega} f \xi \phi^{2}\left(\frac{|y|^{2}}{k^{2}}\right) d x d y-\int_{\Omega} J(\Psi, \xi) \xi \phi^{2}\left(\frac{|y|^{2}}{k^{2}}\right) d x d y \\
-4 \int_{\Omega} \xi \xi_{y} \phi\left(\frac{|y|^{2}}{k^{2}}\right) \phi^{\prime}\left(\frac{|y|^{2}}{k^{2}}\right) \frac{y}{k^{2}} d x d y+\frac{C}{k^{2}}\|\xi\|^{2}+C_{1} \epsilon .
\end{array}
$$

By similar arguments used in (2.50), after detailed calculations, we find that for $k \geq k_{2}$ and $t \geq T_{1}$, the right-hand side of (2.62) is bounded by

$$
C \epsilon+\frac{C_{1}}{k}+C_{2} \int_{|y| \geq k}|f|^{2} d x d y+\frac{\alpha}{4} \int_{\Omega}|\xi|^{2} \phi^{2}\left(\frac{|y|^{2}}{k^{2}}\right) d x d y,
$$

and hence there is $k_{3}>0$ such that for all $k \geq k_{3}$ and $t \geq T_{1}$,

$$
\frac{d}{d t} \int_{\Omega}|\xi|^{2} \phi^{2}\left(\frac{|y|^{2}}{k^{2}}\right) d x d y+\frac{\alpha}{2} \int_{\Omega}|\xi|^{2} \phi^{2}\left(\frac{|y|^{2}}{k^{2}}\right) d x d y \leq C \epsilon .
$$

By Gronwall lemma, we find that for any $k \geq k_{3}$,

$$
\begin{gathered}
\int_{\Omega}|\xi|^{2} \phi^{2}\left(\frac{|y|^{2}}{k^{2}}\right) d x d y \leq e^{-\frac{\alpha}{2}\left(t-T_{1}\right)} \int_{\Omega}\left|\xi\left(T_{1}\right)\right|^{2} \phi^{2}\left(\frac{|y|^{2}}{k^{2}}\right) d x d y+C \epsilon \\
\leq e^{-\frac{\alpha}{2}\left(t-T_{1}\right)}\left\|\xi\left(T_{1}\right)\right\|^{2}+C \epsilon \leq C_{1} e^{-\frac{\alpha}{2}\left(t-T_{1}\right)}+C \epsilon \leq 2 C \epsilon
\end{gathered}
$$

for any $t \geq T_{2}=T_{1}-\frac{2}{\alpha} \ln \frac{C \epsilon}{C_{1}}$. By (2.57) and (2.63) we see that for any $k \geq k_{3}$ and $t \geq T_{2}$,

$$
\int_{\Omega} \phi^{2}\left(\frac{|y|^{2}}{k^{2}}\right)\left(|\theta|^{2}+|\xi|^{2}\right) d x d y \leq C \epsilon
$$

and hence for all $k \geq k_{3}$ and $t \geq T_{1}$,

$$
\int_{|y| \geq \sqrt{2 k}}\left(|\theta|^{2}+|\xi|^{2}\right) d x d y \leq \int_{\Omega} \phi^{2}\left(\frac{|y|^{2}}{k^{2}}\right)\left(|\theta|^{2}+|\xi|^{2}\right) d x d y \leq C \epsilon,
$$

which implies Lemma 2.5. The proof is complete. 


\section{Existence of Global Attractors}

In this section, we prove the existence of global attractors for problem (2.1) $-(2.3)$ in $L^{2}(\Omega) \times$ $L^{2}(\Omega)$. To this end, we need to establish the asymptotic compactness of the solution operator which is stated as follows.

Lemma 3.1. The dynamical system $\{S(t)\}_{t \geq 0}$ is asymptotically compact in $L^{2}(\Omega) \times L^{2}(\Omega)$, i.e., if $t_{n} \rightarrow \infty$ and $\left\{\left(\xi_{0, n}, \theta_{0, n}\right)\right\}_{n=1}^{\infty}$ is bounded in $L^{2}(\Omega) \times L^{2}(\Omega)$, then the sequence $\left\{S\left(t_{n}\right)\left(\xi_{0, n}, \theta_{0, n}\right)\right\}_{n=1}^{\infty}$ has a convergent subsequence.

Proof. Since $\left\{\left(\xi_{0, n}, \theta_{0, n}\right)\right\}_{n=1}^{\infty}$ is bounded in $L^{2}(\Omega) \times L^{2}(\Omega)$, there is $R>0$ such that

$$
\left\|\xi_{0, n}\right\|+\left\|\theta_{0, n}\right\| \leq R, \quad \forall n \in \mathbb{Z}^{+}
$$

By Lemma 2.3, there is a positive number $M$, depending on $\left(\Omega, P_{r}, R_{a}\right)$, such that for every $\left(\xi_{0}, \theta_{0}\right) \in L^{2}(\Omega) \times L^{2}(\Omega)$ with $\left\|\xi_{0}\right\|+\left\|\theta_{0}\right\| \leq R$, the following holds

$$
\left\|S(t)\left(\xi_{0}, \theta_{0}\right)\right\|_{H_{0}^{1}(\Omega) \times H_{0}^{1}(\Omega)} \leq M, \quad \forall t \geq T_{1}
$$

where $T_{1}$ depends on $\left(\Omega, P_{r}, R_{a}\right)$ and $R$. Since $t_{n} \rightarrow \infty$, there is $N_{1}>0$ such that $t_{n} \geq T_{1}$ for all $n \geq N_{1}$. Therefore we have, for $n \geq N_{1}$,

$$
\left\|S\left(t_{n}\right)\left(\xi_{0, n}, \theta_{0, n}\right)\right\|_{H_{0}^{1}(\Omega) \times H_{0}^{1}(\Omega)} \leq M
$$

By (3.3) we find that there is $(\xi, \theta) \in H_{0}^{1}(\Omega) \times H_{0}^{1}(\Omega)$ such that, up to a subsequence,

$$
S\left(t_{n}\right)\left(\xi_{0, n}, \theta_{0, n}\right) \rightarrow(\xi, \theta) \quad \text { in } \quad L^{2}(\Omega) \times L^{2}(\Omega) \quad \text { and } \quad H_{0}^{1}(\Omega) \times H_{0}^{1}(\Omega)
$$

Given $\epsilon>0$, by Lemma 2.5, there are positive numbers $k_{1}$ and $T_{2}$ such that for any $k \geq k_{1}$ and $t \geq T_{2}, S(t)\left(\xi_{0}, \theta_{0}\right)$, with $\left\|\left(\xi_{0}, \theta_{0}\right)\right\| \leq R$, satisfies

$$
\int_{\Omega \backslash \Omega_{k}}\left(\left|S(t) \xi_{0}\right|^{2}+\left|S(t) \theta_{0}\right|^{2}\right) d x d y \leq \frac{\epsilon}{5} .
$$

Let $N_{2}$ be large enough such that $t_{n} \geq T_{2}$ for all $n \geq N_{2}$. Then by (3.5) we obtain, for $n \geq N_{2}$,

$$
\int_{\Omega \backslash \Omega_{k}}\left(\left|S\left(t_{n}\right) \xi_{0, n}\right|^{2}+\left|S\left(t_{n}\right) \theta_{0, n}\right|^{2}\right) d x d y \leq \frac{\epsilon}{5} .
$$


Notice that (3.3) implies that the sequence $\left\{\left.S\left(t_{n}\right)\left(\xi_{0, n}, \theta_{0, n}\right)\right|_{\Omega_{k}}\right\}_{n=1}^{\infty}$ is bounded in $H^{1}\left(\Omega_{k}\right) \times$ $H^{1}\left(\Omega_{k}\right)$ and hence precompact in $L^{2}\left(\Omega_{k}\right) \times L^{2}\left(\Omega_{k}\right)$. Therefore, there is $(\tilde{\xi}, \tilde{\theta}) \in L^{2}\left(\Omega_{k}\right) \times L^{2}\left(\Omega_{k}\right)$ such that, up to a subsequence,

$$
S\left(t_{n}\right)\left(\xi_{0, n}, \theta_{0, n}\right) \longrightarrow(\tilde{\xi}, \tilde{\theta}) \quad \text { in } \quad L^{2}\left(\Omega_{k}\right) \times L^{2}\left(\Omega_{k}\right)
$$

By (3.4) and (3.7), we find that

$$
(\tilde{\xi}, \tilde{\theta})=\left.(\xi, \theta)\right|_{\Omega_{k}},
$$

which means that for every $k \geq k_{1}$,

$$
\left.\left.S\left(t_{n}\right)\left(\xi_{0, n}, \theta_{0, n}\right)\right|_{\Omega_{k}} \longrightarrow(\xi, \theta)\right|_{\Omega_{k}} \quad \text { in } \quad L^{2}\left(\Omega_{k}\right) \times L^{2}\left(\Omega_{k}\right)
$$

In other words, for the given $\epsilon>0$, there is $N_{3}>0$ such that for all $k \geq k_{1}$ and $n \geq N_{3}$,

$$
\int_{\Omega_{k}}\left(\left|S\left(t_{n}\right) \xi_{0, n}-\xi\right|^{2}+\left|S\left(t_{n}\right) \theta_{0, n}-\theta\right|^{2}\right) d x d y \leq \frac{\epsilon}{5}
$$

Since $\xi$ and $\theta$ are in $L^{2}(\Omega)$, there is $k_{2}>0$ such that for all $k \geq k_{2}$,

$$
\int_{\Omega \backslash \Omega_{k}}\left(|\xi|^{2}+|\theta|^{2}\right) d x d y \leq \frac{\epsilon}{5} .
$$

Let $k_{0}=\max \left\{k_{1}, k_{2}\right\}$ and $N_{0}=\max \left\{N_{1}, N_{2}, N_{3}\right\}$, then for all $n \geq N$, we have

$$
\begin{aligned}
& \int_{\Omega}\left(\left|S\left(t_{n}\right) \xi_{0, n}-\xi\right|^{2}+\left|S\left(t_{n}\right) \theta_{0, n}-\theta\right|^{2}\right) d x d y \\
= & \int_{\Omega_{k_{0}}}\left(\left|S\left(t_{n}\right) \xi_{0, n}-\xi\right|^{2}+\left|S\left(t_{n}\right) \theta_{0, n}-\theta\right|^{2}\right) d x d y \\
+ & \int_{\Omega \backslash \Omega_{k_{0}}}\left(\left|S\left(t_{n}\right) \xi_{0, n}-\xi\right|^{2}+\left|S\left(t_{n}\right) \theta_{0, n}-\theta\right|^{2}\right) d x d y \\
\leq & \int_{\Omega_{k_{0}}}\left(\left|S\left(t_{n}\right) \xi_{0, n}-\xi\right|^{2}+\left|S\left(t_{n}\right) \theta_{0, n}-\theta\right|^{2}\right) d x d y \\
+ & 2 \int_{\Omega \backslash \Omega_{k_{0}}}\left(\left|S\left(t_{n}\right) \xi_{0, n}\right|^{2}+\left|S\left(t_{n}\right) \theta_{0, n}\right|^{2}\right) d x d y \\
+ & 2 \int_{\Omega \backslash \Omega_{k_{0}}}\left(|\xi|^{2}+|\theta|^{2}\right) d x d y \leq \epsilon
\end{aligned}
$$

where the last inequality is obtained by (3.6), (3.9) and (3.10). Notice that (3.11) shows that

$$
S\left(t_{n}\right)\left(\xi_{0, n}, \theta_{0, n}\right) \longrightarrow(\xi, \theta) \quad \text { in } \quad L^{2}(\Omega) \times L^{2}(\Omega)
$$

and hence $\{S(t)\}_{t \geq 0}$ is asymptotically compact. The proof is complete. 
We are, now, ready to prove the existence of a global attractor for problem (2.1)-(2.3).

Theorem 3.2. Problem (2.1)-(2.3) has a global attractor $\mathcal{A}$ in $L^{2}(\Omega) \times L^{2}(\Omega)$, which is compact, invariant and attracts every bounded set with respect to the norm of $L^{2}(\Omega) \times L^{2}(\Omega)$.

Proof. By Lemma 2.2 , the dynamical system $\{S(t)\}_{t \geq 0}$ has a bounded absorbing set in $L^{2}(\Omega) \times$ $L^{2}(\Omega)$, and by Lemma 3.1, $\{S(t)\}_{t \geq 0}$ is asymptotically compact. Then the existence of a global attractor follows immediately from the standard attractor theory (see e.g., [4, 5, 13, 21, 23]).

\section{Regularity of Global Attractors}

In this section, we investigate the regularity of the global attractor obtained in Theorem 3.2. We will show that the global attractor $\mathcal{A}$ is actually contained in a bounded subset of $H^{2}(\Omega) \times H^{2}(\Omega)$. We start with the following lemma

Lemma 4.1. Suppose that $\left(\xi_{0}, \theta_{0}\right) \in L^{2}(\Omega) \times L^{2}(\Omega)$. Then the solution $(\xi, \theta)$ of problem (2.1)(2.3) satisfies

$$
\left\|\frac{d \xi}{d t}\right\|+\left\|\frac{d \theta}{d t}\right\| \leq M, \quad \forall t \geq T
$$

where $M$ depends only on the data $\left(\Omega, P_{r}, R_{a}\right), T$ depends on the data $\left(\Omega, P_{r}, R_{a}\right)$ and $R$ when $\left\|\xi_{0}\right\| \leq R$ and $\left\|\theta_{0}\right\| \leq R$

Proof. By (2.8) and (2.1) we find that

$$
\begin{aligned}
\left\|\frac{d \xi}{d t}\right\| & \leq\|\triangle \xi\|+\|J(\psi, \xi)\|+C\|\nabla \theta\|+\|f\| \\
& \leq\|\triangle \xi\|+C\|\xi\|\|\triangle \xi\|+C_{1}\|\nabla \theta\|+C_{2} \leq C\|\triangle \xi\|+C_{1}, \quad \forall t \geq T,
\end{aligned}
$$

where the last inequality is obtained by Lemma 2.3. By (4.1) and Lemma 2.3 again we get, for $t \geq T$

$$
\int_{t}^{t+1}\left\|\frac{d \xi}{d t}\right\|^{2} d t \leq C \int_{t}^{t+1}\|\triangle \xi\|^{2} d t+C_{1} \leq C .
$$

Similarly, by (2.3), we find that, for $t \geq T$,

$$
\left\|\frac{d \theta}{d t}\right\| \leq C\|\triangle \theta\|+\|J(\psi, \xi)\|+\|g\| \leq C\|\triangle \theta\|+C_{1},
$$


which along with Lemma 2.3 implies that, for $t \geq T$,

$$
\int_{t}^{t+1}\left\|\frac{d \theta}{d t}\right\|^{2} d t \leq C \int_{t}^{t+1}\|\triangle \theta\|^{2} d t+C_{1} \leq C .
$$

Let $\tilde{\xi}=\frac{d \xi}{d t}$ and $\tilde{\theta}=\frac{d \theta}{d t}$. Then it follows from (4.2) and (4.4) that, for $t \geq T$,

$$
\int_{t}^{t+1}\left(\|\tilde{\xi}(t)\|^{2}+\|\tilde{\theta}(t)\|^{2}\right) d t \leq C .
$$

We now differentiate (2.1) and (2.3) with respect to $t$ to obtain

$$
\frac{\partial \tilde{\xi}}{\partial t}-\triangle \tilde{\xi}+J\left(\Psi_{t}, \xi\right)+J(\Psi, \tilde{\xi})+\frac{R_{a}}{P_{r}} \frac{\partial \tilde{\theta}}{\partial x}=0
$$

and

$$
\frac{\partial \tilde{\theta}}{\partial t}-\frac{1}{P_{r}} \triangle \tilde{\theta}+J\left(\Psi_{t}, \theta\right)+J(\Psi, \tilde{\theta})=0 .
$$

Taking the inner product of (4.6) with $\tilde{\xi}$ in $L^{2}(\Omega)$, we find that

$$
\frac{1}{2} \frac{d}{d t}\|\tilde{\xi}\|^{2}+\|\nabla \tilde{\xi}\|^{2}+\left(J\left(\Psi_{t}, \xi\right), \tilde{\xi}\right)+\frac{R_{a}}{P_{r}}\left(\frac{\partial \tilde{\theta}}{\partial x}, \tilde{\xi}\right)=0
$$

By (2.8) we have

$$
\left|\left(J\left(\Psi_{t}, \xi\right), \tilde{\xi}\right)\right| \leq\left\|J\left(\Psi_{t}, \xi\right) \mid\right\|\|\tilde{\xi}\| \leq C\|\tilde{\xi}\|^{2}\|\triangle \xi\| \leq\|\tilde{\xi}\|^{4}+C\|\triangle \xi\|^{2}
$$

We also have the following inequality

$$
\left|\frac{R_{a}}{P_{r}}\left(\frac{\partial \tilde{\theta}}{\partial x}, \tilde{\xi}\right)\right| \leq C\|\nabla \tilde{\theta}\|\|\tilde{\xi}\| \leq \frac{1}{2 P_{r}}\|\nabla \tilde{\theta}\|^{2}+C\|\tilde{\xi}\|^{2} \leq \frac{1}{2 P_{r}}\|\nabla \tilde{\theta}\|^{2}+\|\tilde{\xi}\|^{4}+C_{1} .
$$

It follows from (4.8)-(4.10) that

$$
\frac{d}{d t}\|\tilde{\xi}\|^{2}+\|\nabla \tilde{\xi}\|^{2} \leq C\|\tilde{\xi}\|^{4}+C_{1}\|\triangle \xi\|^{2}+\frac{1}{2 P_{r}}\|\nabla \tilde{\theta}\|^{2}+C_{2} .
$$

Now, by taking the inner product of (4.7) with $\tilde{\theta}$ in $L^{2}(\Omega)$ we get

$$
\frac{1}{2} \frac{d}{d t}\|\tilde{\theta}\|^{2}+\frac{1}{P_{r}}\|\nabla \tilde{\theta}\|^{2}=-\left(J\left(\Psi_{t}, \theta\right), \tilde{\theta}\right) .
$$

By an argument similar to (4.9), the right-hand side of (4.12) is bounded by

$$
\left|\left(J\left(\Psi_{t}, \theta\right), \tilde{\theta}\right)\right| \leq\|\tilde{\xi}\|^{4}+\|\tilde{\theta}\|^{4}+C\|\triangle \theta\|^{2} .
$$


By (4.12) and (4.13) we find that

$$
\frac{d}{d t}\|\tilde{\theta}\|^{2}+\frac{1}{P_{r}}\|\nabla \tilde{\theta}\|^{2} \leq 2\|\tilde{\xi}\|^{4}+2\|\tilde{\theta}\|^{4}+C\|\triangle \theta\|^{2} .
$$

By (4.11) and (4.14) it follows that

$$
\begin{gathered}
\frac{d}{d t}\left(\|\tilde{\xi}\|^{2}+\|\tilde{\theta}\|^{2}\right)+\|\nabla \tilde{\xi}\|^{2}+\frac{1}{2 P_{r}}\|\nabla \tilde{\theta}\|^{2} \\
\leq C\left(\|\tilde{\xi}\|^{4}+\|\tilde{\theta}\|^{4}\right)+C_{1}\left(1+\|\triangle \xi\|^{2}+\|\triangle \theta\|^{2}\right),
\end{gathered}
$$

which implies that

$$
\begin{aligned}
\frac{d}{d t}\left(\|\tilde{\xi}\|^{2}+\|\tilde{\theta}\|^{2}\right) & \leq C\left(\|\tilde{\xi}\|^{2}+\|\tilde{\theta}\|^{2}\right)\left(\|\tilde{\xi}\|^{2}+\|\tilde{\theta}\|^{2}\right) \\
& +C_{1}\left(1+\|\triangle \xi\|^{2}+\|\triangle \theta\|^{2}\right) .
\end{aligned}
$$

By Lemma 2.3, (4.5) and the uniform Gronwall inequality we finally obtain that

$$
\|\tilde{\xi}(t)\|^{2}+\|\tilde{\theta}(t)\|^{2} \leq C, \quad \forall t \geq T+1
$$

which concludes the proof.

Lemma 4.2. Suppose that $\left(\xi_{0}, \theta_{0}\right) \in L^{2}(\Omega) \times L^{2}(\Omega)$. Then the solution $(\xi, \theta)$ of problem (2.1)(2.3) satisfies

$$
\|\xi(t)\|_{H^{2}}+\|\theta(t)\|_{H^{2}} \leq M, \quad \forall t \geq T
$$

where $M$ depends only on the data $\left(\Omega, P_{r}, R_{a}\right), T$ depends on the data $\left(\Omega, P_{r}, R_{a}\right)$ and $R$ when $\left\|\xi_{0}\right\| \leq R$ and $\left\|\theta_{0}\right\| \leq R$

Proof. By (2.1) we have the following inequality

$$
\begin{aligned}
\|\triangle \xi\| & \leq\left\|\frac{\partial \xi}{\partial t}\right\|+\|J(\psi, \xi)\|+C\|\nabla \theta\|+\|f\| \leq C+C_{1}\|\Psi\|_{H^{3}}\|\nabla \xi\| \\
& \leq C+C_{1}\|\nabla \xi\|^{2} \leq C,
\end{aligned}
$$

where we have used (2.9) and Lemmas 2.3 and 4.1. Similarly, by (2.1) we see that

$$
\|\triangle \theta\| \leq\left\|\frac{\partial \theta}{\partial t}\right\|+\|J(\psi, \xi)\|+\|g\| \leq C .
$$

From (4.18) and (4.19), Lemma 4.2 follows. 
We are now in position to prove the regularity of the global attractor in $H^{2}(\Omega) \times H^{2}(\Omega)$.

Theorem 4.3. The global attractor $\mathcal{A}$ obtained in Theorem 3.2 is bounded in $H^{2}(\Omega) \times H^{2}(\Omega)$, i.e., there is a positive constant $M$ such that

$$
\|(\xi, \theta)\|_{H^{2}(\Omega) \times H^{2}(\Omega)} \leq M, \quad \forall(\xi, \theta) \in A .
$$

Proof. Since $A$ is bounded in $L^{2}(\Omega) \times L^{2}(\Omega)$, by Lemma 4.2, there is a bounded set $E \subseteq$ $H^{2}(\Omega) \times H^{2}(\Omega)$ and $T>0$ such that

$$
S(t) A \subseteq E, \quad \forall t \geq T
$$

But $S(t) A \subseteq A$ and hence $A \subseteq E$. The proof is complete.

\section{References}

[1] F. Antoci and M. Prizzi, Reaction-Diffusion equations on unbounded thin domains, Topological Methods in Nonlinear Analysis, 18 (2001), 283-302.

[2] F. Antoci and M. Prizzi, Attractors and global averaging of non-autonomous ReactionDiffusion equations in $\mathbb{R}^{n}$. Topological Methods in Nonlinear Analysis, 20 (2002), 229-259.

[3] J. M. Arrieta, J. W. Cholewa, T. Dlotko, and A. Rodriguez-Bernal, Asymptotic behavior and attractors for Reaction Diffusion equations in unbounded domains, Nonlinear Analysis, 56 (2004), 515-554.

[4] A.V. Babin and M.I. Vishik, Attractors of Evolution Equations, North-Holland, Amsterdam, 1992.

[5] J.M. Ball, Continuity properties and global attractors of generalized semiflows and the Navier-Stokes equations, J. Nonl. Sci., 7 (1997), 475-502.

[6] J.M. Ball, Global attractors for damped semilinear wave equations, Discrete Contin. Dyn. Syst. 10 (2004), 31-52. 
[7] S. Chen, Symmetry analysis of convection on patterns, Comm. Theor. Phys., 1 (1982), 413-426.

[8] M. J. Feigenbaum, The onset spectrum of turbulence, Phys. Lett. A, 74 (1979), 375-378.

[9] B. Guo, Spectral method for solving the two-dimensional New-Boussinesq equations, Acta. Math. Appl., 5 (1989), 208-218.

[10] B. Guo, Nonlinear Galerkin methods for solving the two-dimensional New-Boussinesq equations, Advance in Math., 22 (1993), 179-181.

[11] B. Guo and B. Wang, Gevrey class regularity and approximate inertial manifolds for the New-Boussinesq equations, Chin. Ann. of Math., 19B (1998), 179-188.

[12] B. Guo and B. Wang, Approximate inertial manifolds for the two-dimensional NewBoussinesq equations, J. Partial Diff. Equ., 9 (1996), 237-250.

[13] J.K. Hale, Asymptotic Behavior of Dissipative Systems, American Mathematical Society, Providence, RI, 1988.

[14] N. Ju, The $H^{1}$-compact global attractor for the solutions to the Navier-Stokes equations in two-dimensional unbounded domains, Nonlinearity, 13 (2000), 1227-1238.

[15] F. Morillas and J. Valero, Attractors for Reaction-Diffusion equations in $\mathbb{R}^{n}$ with continuous nonlinearity, Asymptotic Analysis, 44 (2005), 111-130.

[16] I. Moise, R. Rosa and X. Wang, Attractors for non-compact semigroups via energy equations, Nonlinearity, 11 (1998), 1369-1393.

[17] M. Prizzi, Averaging, Conley index continuation and recurrent dynamics in almostperiodic equations, J. Differential Equations, 210 (2005), 429-451.

[18] J.C. Robinson, Infinite-Dimensional Dynamical Systems, Cambridge University Press, Cambridge, UK, 2001. 
[19] A. Rodrigue-Bernal and B. Wang, Attractors for partly dissipative Reaction Diffusion systems in $\mathbb{R}^{n}$, J. Math. Anal. Appl., 252 (2000), 790-803.

[20] R. Rosa, The global attractor for the 2D Navier-Stokes flow on some unbounded domains, Nonlinear Anal., 32 (1998), 71-85.

[21] R. Sell and Y. You, Dynamics of Evolutionary Equations, Springer-Verlag, New York, 2002.

[22] C. Sun and C. Zhong, Attractors for the semilinear Reaction-Diffusion equation with distribution derivatives in unbounded domains, Nonlinear Analysis, 63 (2005), 49-65.

[23] R. Temam, Infinite-Dimensional Dynamical Systems in Mechanics and Physics, SpringerVerlag, New York, 1997.

[24] B. Wang, Attractors for reaction-diffusion equations in unbounded domains, Physica D, 128 (1999), 41-52. 